\title{
Fascite Nodular: Relato de Caso Clínico com Estudo Imunohistoquímico
} Nodular Fasciitis: Case Report with Immunohistochemical Analisys

\author{
Pantelis Varvaki Rados* \\ Manoel Sant'Ana Filho* \\ Isabel da Silva Lauxen** \\ João Batista Burzlaff*** \\ Vinicius Coelho Carrard***
}

\section{RESUMO}

A fascite nodular é uma lesão benigna de células fusiformes que freqüentemente é confundida com lesões malignas. 0 relato do caso clínico coincide com os aspectos clínicos, microscópicos e imunohistoquímicos. 0s autores salientam que a definição do diagnóstico desta lesão deve ser realizado a partir de suas características microscópicas que orientam a definição do painel imunohistoquímico.

\section{PALAVRAS-CHAVE}

Fascite nodular, cavidade bucal, lesões de células fusiformes

\section{INTRODUÇÃO E REVISÃO DE LITE- RATURA}

A fasciite nodular é uma lesão benigna, que se caracteriza pela proliferação de fibroblastos, miofibroblastos e histiócitos. Se desenvolve geralmente junto a inserções musculares e sua etiologia é desconhecida, apesar de, em alguns casos, ser relatado trauma prévio (PRICE, SILLIPHANT, SHUMAN, 1961; BERNSTEIN, LATTES, 1982) . Clinicamente, apresenta-se como uma lesão nodular, submucosa, firme a palpação, de crescimento rápido, sendo, muitas vezes, confundida com lesões malignas (PRICE; SILLIPHANT; SHUMAN, 1961; HUTTER et al, 1962; RAKOWER, 1971; CHUNG; ENZINGER, 1975; CHARTIER; ALLAIRE; TOSCANO, 1994; SHLOMI et al, 1994). Na cavidade bucal, a localização mais comumente afetada é a mucosa jugal (SATO et al, 1981; LUMERMAN; BODNER; ZAMBITO, 1972; SHLOMI et al, 1994). As lesões normalmente não ultrapassam $2 \mathrm{~cm}$ de diâmetro e, durante a enucleação cirúrgica mostra-se firmemente aderida, não sendo observada recorrência (CULBERSON; ENTERLINE, 1960; PRICE; SILLIPHANT; SHUMAN, 1961; HUTTER et al, 1962; CHUNG; ENZINGER, 1975; SATO et al, 1981; BERNSTEIN; LATTES, 1982). Alguns autores relatam que a lesão pode involuir espontaneamente (CHARTIER; ALLAIRE; TOSCANO, 1994).

Microscopicamente caracteriza-se por proliferação de células fusiformes formando feixes curtos, dispostos ao acaso ou formando nódulos. A lesão é bem circunscrita, mas não encapsulada, infiltrando-se, através de projeções digitiformes, no tecido muscular ou adiposo adjacente. Misturando-se com essas células fusiformes, pode existir um tecido mixóide ou fibras delicadas de colágeno. A lesão apresenta capilares imaturos, com células endoteliais proeminentes e arranjadas radial ou paralelamente. Encontram-se ainda células inflamatórias e hemácias extravasadas, mas sem a presença de hemossiderina. Mitoses, células gigantes, material mucóide e fibrina podem estar presentes (KONWALER, 1955; CULBERSON; ENTERLINE, 1960 ; PRICE; SILLIPHANT; SHUMAN, 1961; HUTTER et al, 1962; RAKOWER, 1971; CHUNG; ENZINGER; 1975; CHARTIER; ALLAIRE; TOSCANO, 1994; EVERSOLE et al 1999, MARTINEZ-BLANCO et al, 2000).

O rápido crescimento, a alta celularidade e o comportamento infiltrativo dessa lesão sugerem malignidade, sendo necessário o seu conhecimento, a fim de evitar uma cirurgia radical desnecessária (LUMERMAN; BODNER; ZAMBITO, 1972 ; CHARTIER; ALLAIRE; TOSCANO, 1994).

Em função da dificuldade para estabelecer o diagnóstico, pode-se utilizar a técnica imunohistoquímica como um recurso auxiliar no diagnóstico, a fim de minimizar a chance de erro (MONTGOMERY, MEIS, 1991; SHLOMI et al, 1994; EVERSOLE et al, 1999). Por outro lado, alguns autores consideram que este recurso desnecessário, acreditando que os achados morfológicos suficientes para tanto (CHARTIER, ALLAIRE, TOSCANO, 1994).

O objetivo deste trabalho é fazer o relato de um caso clínico de fascite nodular, no qual foi utilizada a técnica imunohistoquímica para auxiliar no estabelecimento do diagnóstico.

\section{RELATO DE CASO}

Paciente C.P.S., sexo feminino, 41 anos, raça branca, apresentou lesão palpável na região do músculo masseter, lado direito, com $1 \mathrm{~cm}$ de diâmetro, coloração azulada, com 4 meses de evolução. 0 exame ultrassonográfico mostrou nódulo hipogênico bem delimitado junto ao masseter. Com base nos achados clínico e ultrassonográfico, que indicavam que a lesão era bem delimitada, procedeu-se com a biópsia parcial da lesão. 0 exame histopatológico mostrou proliferação de células fusiformes formando feixes com discreta atipia (figura 1), áreas com presença de células inflamatórias e hemorragia (figura 2). A presença de um pequeno número de figuras de mitose associado à ausência de pleomorfismo celular descartou a possibilidade de uma lesão maligna, sendo o diagnóstico inicial lesão benigna de células fusiformes (figuras 1 e 2). Tendo em vista que os aspectos morfológicos não permitiram a definição da natureza das células presentes nessa lesão, optou-se pela utilização de uma técnica auxiliar, buscando-se um diagnóstico definitivo. Para o estabelecimento do diagnóstico definitivo o material foi submetido a um painel imunohistoquímico, utilizando-se os marcadores: anti-vimentina (células conjuntivas), anti-S100 (células nervosas), anti-desmina (células musculares), alfa anti-CD 68 (histiócitos), anti-HHF (miofibroblastos), obtendo-se o seguinte resultado: 


\begin{tabular}{|cc|}
\hline Marcador & Marcação \\
\hline Vimentina & + \\
S100 & - \\
HHF35 35 & + \\
Desmina & - \\
CD 68 & + \\
\hline
\end{tabular}

0 resultado do painel imunohistoquímico definiu a lesão como fascí́te nodular (figura 3), sendo realizada a sua enucleação como tratamento definitivo.

\section{DISCUSSÃ O}

O caso relatado apresentava características clínicas usualmente descritas na literatura como: crescimento rápido, localização submucosa e próxima a inserções musculares. Algumas informações importantes, como aderência da lesão aos planos musculares (HUTTER et al, 1962), não foram percebidas pelo cirurgião, o que poderia contribuir no estabelecimento do diagnóstico, na medida em que essa lesão parece crescer a partir da fáscia do tecido muscular.

0 resultado do exame ultrassonográfico, que definiu a lesão como bem delimitada, sugeriu a natureza benigna da lesão, porém o seu rápido crescimento não permitia descartar um comportamento mais agressivo.

Nos casos descritos na literatura observa-se que, por muito tempo, o diagnóstico desse tipo de lesão era definido exclusivamente a partir de suas características microscópicas. Por outro lado, pode-se constatar que, em diversas situações, isso determinou diagnósticos incorretos, levando a tratamentos excessivamente radicais (BERNSTEIN; LATTES, 1982). Em função disso, considera-se o emprego de marcadores imunohistoquímicos bastante útil (MONTGOMERY; MEIS, 1991), ao contrário do que afirmam alguns autores (ALLARE; CHARTIER; TOSCANO, 1991), principalmente em função dos diferentes padrões histológicos que a lesão pode apresentar (PRICE; SILLIPHANT; SHUMAN, 1961). Destaca-se a importância da consideração do arranjo estrutural e da morfologia nuclear das células presentes nessas lesões que, juntamente com o resultado do painel imunohistoquímico, determinam um diagnóstico preciso e um tratamento correto (MONTGOMERY; MEIS, 1991).

Com base nos achados microscópicos observados pela técnica da HematoxilinaEosina foi possível definir-se a natureza benigna da lesão e sua origem mesenquimal. Considerando que a lesão não mostrava um pleomorfismo celular evidente e raramente observavam-se mitoses, a possibilidade de lesão maligna foi descartada. Além disso, a presença de hemácias extravasadas e células inflamatórias dispersas por toda a lesão foram características que direcionaram o diagnóstico para fascite nodular (KONWALER et al , 1955; PRICE; SILLIPHANT; SHUMAN, 1961; CHUNG; ENZINGER, 1975; SATO et al, 1981; ALKAN; GÜNHAN; ÇELENK, 2001; MARTINEZ-BLANCO et al, 2002). Essa característica parece ser resultado da fragilidade capilar típica dessa lesão, uma vez que usualmente encontra-se neoformação vascular, e não provocada pelo ato cirúrgico como sugerem CULBERSON; ENTERLINE (1960). Caso isso fosse verdadeiro, não seriam observadas essas alterações em áreas distantes da zona da incisão (figura 2). Uma vez que, além da neoformação vascular, observa-se, muitas vezes, a presença de áreas mucóides, sugerindo fibrina, encontrase reforçada a hipótese de essa lesão se tratar de um processo reacional (PRICE; SILLIPHANT; SHUMAN, 1961; CHUNG; ENZINGER, 1975; BERNSTEIN; LATTES, 1982; MONTGOMERY; MEIS, 1991).

O arranjo em feixes curtos de células fusiformes e a presença de áreas mixóides são outros aspectos importantes sendo características diferenciais dessa lesão em relação às outras lesões de células fusiformes (PRICE; SILLIPHANT; SHUMAN, 1961; HUTTER et al, 1962; BERNSTEIN; LATTES, 1982 ALLAIRE; CHARTIER; TOSCANO, 1991 ; MARTINEZ-BLANCO et al, 2002).

Diante da dificuldade que esta lesão impõe quanto ao estabelecimento do diagnóstico algumas características devem ser observadas. Embora possa existir um pleomorfismo celular nessas lesões, este se restringe a forma e tamanho das células, o hipercromatismo normalmente não ocorre, sendo uma característica observada em histiocitomas fibrosos. Figuras de mitose, quando encontradas, apresentam número baixo e aspecto normal, não sendo mais do que três por campo microscópico em grande aumento. Em relação ao infiltrado inflamatório presente na lesão, este normalmente não inclui neutrófilos, sendo os linfócitos as células mais presentes (KAY, 1960; PRICE;
SILLIPHANT; SHUMAN, BERNSTEIN; LATTES, 1982)

Do ponto vista da técnica imunohistoquímica, não foi utilizado o marcador citoqueratina, pois, em nenhum momento se considerou a possibilidade de as células presentes na lesão terem origem epitelial. Além disso, a utilização de mais um marcador imunohistoquímico tornaria o exame ainda mais oneroso para o paciente, sendo esse considerado desnecessário.

\section{ABSTRACT}

Nodular fasciitis is a benign lesion composed of fusiform cells frequently misdiagnosed as malignant lesion. This report of case have similar clinical, microscopic and immunohistochemical with the literature reviewed. The immunohistochemical pannel should be decided upon the microscopic appearence of the lesion.

\section{KEYWORDS}

Nodular fasciitis, oral cavity, spindle cell lesion

\section{REFERENCIAS}

ALKAN, A.; GÜNHAN, Ö.; ÇELENK, P. Oral Nodular Fasciitis: a Case Report. J. Oral Sci., v. 43, n. 3, p. 217-220, Sep. 2001.

BERNSTEIN, K. E.; LATTES, R. Nodular (Pseudosarcomatous) Fasciitis, A Nonrecurrent Lesion: Clinicopathologic Study of 134 Cases. Cancer, v. 49, no ${ }^{\circ}$ 15, p. 1668-1678, Apr. 1982.

CHARTIER, S.; ALLAIRE, G.; TOSCANO, M. Nodular Fasciitis of the Upper Lip Mimicking a Sarcoma. Int. J. Derm., v. 33, $\mathrm{n}^{\circ} .7$, p. 503-504, July 1994.

CHUNG, E.B.; ENZINGER, F. M. Proliferative Fascïtis. Cancer, v. 36, p.14501458, Oct.1975.

CULBERSON, J. D.; ENTERLINE, H. Pseudosarcomatous Fasciitis. A Distinctive Clinical-Pathologic Entity- Report of Five Cases. Ann. Surg., v. 151, nº . 2, p. 235-240, Feb. 1960

EVERSOLE, L. R. et al. Nodular Fasciitis and Solitary Fibrous Tumor of the Oral Region - Tumors of Fibroblast Heterogeneity. Oral Surg., Oral Med., Oral Pathol., v. 87, p. 471-476, Apr.1999.

HUTTER, R. V. P; STEWART, F. W.; FOOTE, F. W. Fasciitis- A Report of 70 Cases with Follow-Up Proving the Benignity of the Lesion. Cancer, v. 15, nº. 5 , p. 992 1003, Sept.Oct. 1962.

HUTTER, R. V. P. et al. Parosteal Fasciitis- A Self-Limited Benign Process that simulates a Malign Neoplasm. Am. J. Surg., v.104, p.800- 
807, Dec. 1962.

JORDAN, R.C.K.; REGEZI, J. A. Oral Spindle Cell Neoplasms: A Review of 307 Cases. Oral Surg., Oral Med., Oral Pathol., v. 95, p. 117-125, June 2003.

KAY, S. Subcutaneous Pseudosarcomatous Fibromatosis Report of 4 Cases. Am. J. Clin Pathol., v.33, $\mathrm{n}^{\circ} .5$, p. $433-438$, May 1960.

KONWALER, B. E.; KEASBEY, L.; KAPLAN, L. Subcutaneous Pseudosarcomatous Fibromatosis (Fasciitis). Am. J. Clin. Pathol., v.25, p. 241-252, Mar. 1955.

LUMERMAN, H.; BODNER, B.; ZAMBITO, R. Intraoral (Submucosal) Pseudosarcomatous Nodular Fasciitis- Report of a case. Oral Surg., v. $34, n^{0} .2$, p. 238-244, Aug. 1972.

MARTÍNEZ-BLANCO, M. et al. Maxillofacial Nodular Fasciitis: a report of 3 Cases. J. Oral Maxillofac. Surg., v. 60, p. 1211-1214, Oct. 2002.

MONTGOMERY, E. A. ; MEIS, J. M. Nodular Fasciitis, its Morphologic Spectrum and Immunohistochemical Profile. Am J. Surg. Pathol., v. 15, nº. 10, p. 942-948, Oct. 1991.

PRICE, E. B. ; SILLIPHANT, W. M. ; SHUMAN, R. Nodular Fasciitis : A Clinicalpathologic Analisys of $65 \mathrm{Ca}-$ ses. Am. J. Clin. Pathol., v.35, $\mathrm{n}^{\circ} .2$, p.122-156, Feb. 1961.

RAKOWER, W. Fasciitis : An Unusual diagnosis, and the Clinician's Dilemma - Report of a Case. J. Oral Surg., v.29, p.503-506, July 1971.

SATO, M. et al. Submcosal Nodular Fasciiitis Arising Within the Buccal Area - Report of a Case. Int. J. Oral Sug., v.10, no. 3, p. 210-213, Jun. 1981.

SHLOMI, B. et al. Immunohistochemical Analisys of a Case of Intraoral Nodular Fasciitis. J. Oral Maxillofac. Surg., v. 52, p. 323-326, Mar 1994.

Endereço para Correspondência: Manoel Sant'Ana Filho

Ramiro Barcelos, 2492

Patologia - Fac. Odontologia da UFRGS

E-mail: manoel@ufrgs.br

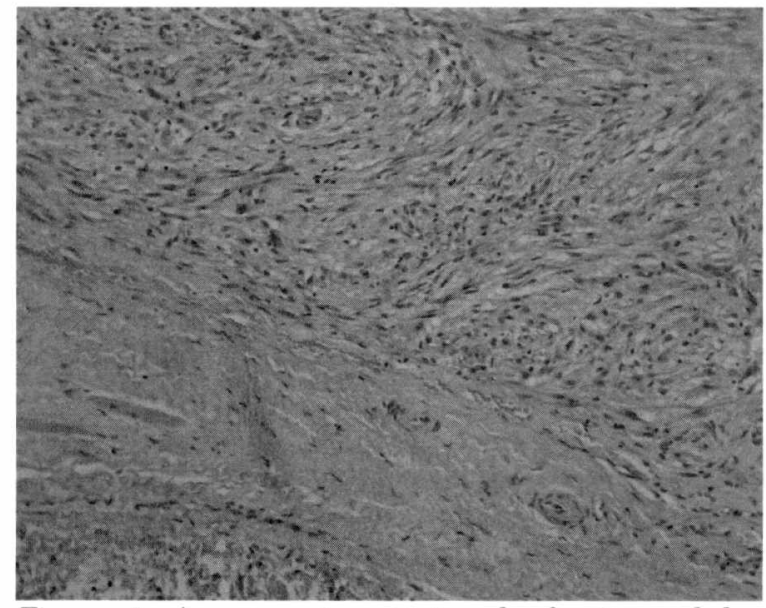

Figura 1. Aspecto microscópico da fascite nodular apresentando porção de tecido conjuntivo que se continua com a proliferação de células fusiformes disposta em feixes curtos e entrecruzados. HE, $100 X$.

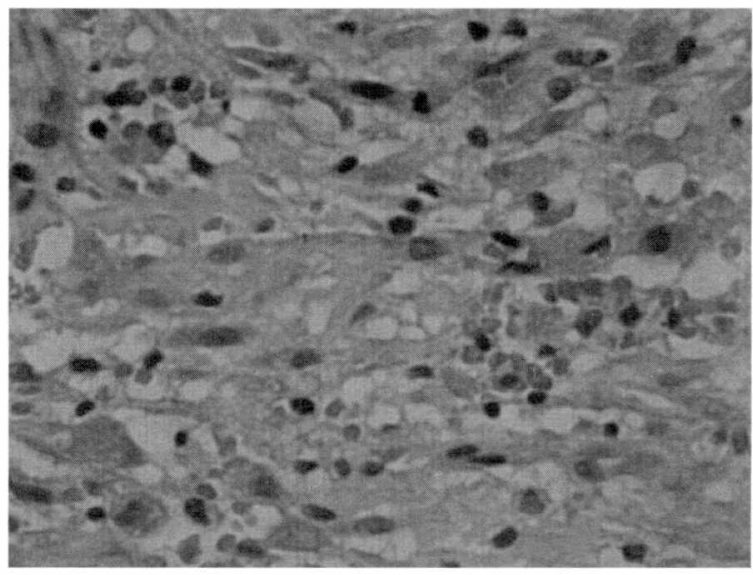

Figura 2. Fotomicrografia mostrando as áreas hemorrágicas e células inflamatórias em meio a proliferação de células fusiformes. $\mathrm{HE}, 400 X$.

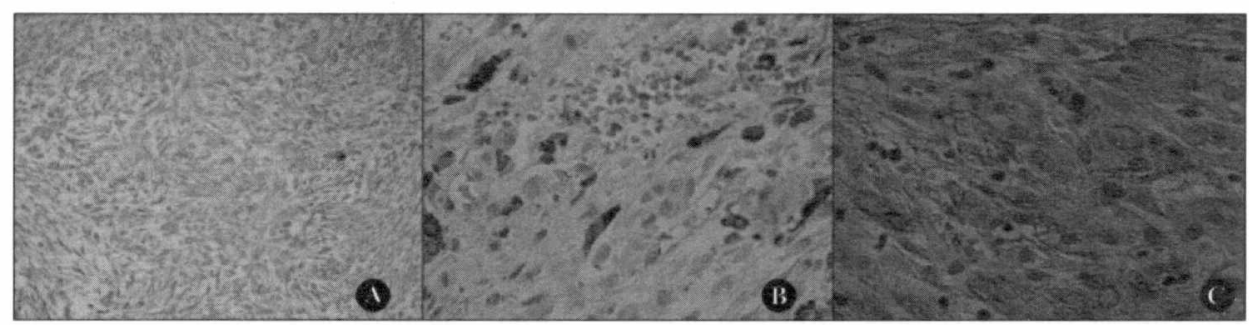

Figura 3. Fotomicrografia em maior aumento mostrando marcação positiva para vimentina (A), CD68 (B) e HHF35 (C), que confirma a linhagem fibrohistiocítica característica da fascite nodular. Diluições: Vimentina, $1: 200(100 x)$; CD68, $1: 10(400 x)$; HHF35, 1 :4000(400x); contra-coloração : Hematoxilina de Harris. 